\title{
Visual Hallucinations Possibly Associated with Clarithromycin Administration at Therapeutic Dosage in Two Children
}

\author{
Nilgun Erkek Saliha Senel Candemir Karacan \\ Department of Pediatrics, Dr. Sami Ulus Children's Hospital, Ankara, Turkey
}

\section{Key Words}

Clarithromycin · Antibiotics · Hallucination · Adverse drug reaction, child

\begin{abstract}
Objective: Our aim was to present 2 children with visual hallucinations possibly associated with clarithromycin administration at therapeutic dosage. Subjects and Methods: Two children were admitted to our hospital with sudden onset of visual hallucinations after taking clarithromycin at therapeutic dosage by mouth. Physical examination, laboratory investigations and imaging studies were normal. The symptoms gradually disappeared once the clarithromycin therapy had been discontinued, making us suspect clarithromycin as the agent responsible for the visual hallucinations. They were observed monthly for a year without any symptoms or further treatment. Conclusion: This report highlights hallucinations due to therapeutic doses of clarithromycin therapy as a possible new side effect in children.
\end{abstract}

Copyright $\odot 2009$ S. Karger AG, Basel
(C) 2009 S. Karger AG, Basel

1011-7571/09/0184-0332\$26.00/0

Fax +4161306 1234

E-Mail karger@karger.ch

www.karger.com
Accessible online at:

www.karger.com/mpp

\section{Introduction}

Clarithromycin is a commonly used advanced-generation macrolide antibiotic. Several adverse reactions have been associated with the ingestion of clarithromycin, and the most frequently reported ones are gastrointestinal in nature $[1,2]$. We describe acute transient visual hallucinations associated with clarithromycin ingestion in 2 children. To our knowledge, hallucinations due to therapeutic doses of clarithromycin therapy in children have not been previously reported in the English pediatric literature, and hallucination may be a new adverse effect profile in children.

\section{Case Reports}

Case 1

A 6-year-old girl was admitted to our hospital with sudden onset of visual hallucinations together with marked fear shortly after taking the second dose of clarithromycin at therapeutic dosage $(15 \mathrm{mg} / \mathrm{kg} /$ day divided every $12 \mathrm{~h})$ by mouth for acute sinusitis. She reported seeing fish, butterflies and spiders in her visual fields. She was fully aware that the images were not real and was able to describe them vividly. She was not having any other medication and had never experienced any adverse reaction to medication. She had no known allergies and no history of medical or 
neuropsychiatric alterations. A detailed family history did not reveal any abnormalities including neuropsychiatric disorders. On physical examination, her blood pressure was $100 / 70 \mathrm{~mm} / \mathrm{Hg}$, the heart rate was 80 beats $/ \mathrm{min}$. She had no fever $\left(36.7^{\circ} \mathrm{C}\right)$, and the cardiac, respiratory and abdominal examinations were normal. There were no focal neurological signs. On mental status examination, she remained cooperative, oriented in space, time and person, but she was afraid of seeing images. Laboratory investigations of serum glucose, electrolyte levels, liver and kidney functions, full blood count, erythrocyte sedimentation rate and C-reactive protein were within normal limits. Cerebrospinal fluid analysis was normal. A toxicology screen including mycotoxins, barbiturates and cholinesterase inhibitors, as well as all cultures were negative. An electroencephalogram was unremarkable. Cranial magnetic resonance imaging did not reveal any abnormalities. Clarithromycin was suspected as the agent responsible for the hallucinations and was therefore discontinued. Over the following $24 \mathrm{~h}$, the visual hallucinations gradually decreased. Neurologic and psychiatric evaluations did not reveal findings consistent with a psychotic disorder or a neurological impairment. On hospital day 2, she continued to improve and was released on hospital day 3 when she had completely recovered from the hallucinations. The clarithromycin treatment was substituted with amoxycillin clavulanic acid. She was observed over the following year without hallucinations or any other neuropsychiatric symptoms.

Case 2

A 7-year-old boy was admitted with the complaint of seeing spiders in his visual fields. He had been taking clarithromycin at therapeutic dosage for acute otitis media for 5 days. He had no other medication and no known allergies. His health history and family history were unremarkable. Physical examination was normal except for the hallucinations. Serum biochemistry tests, full blood count, erythrocyte sedimentation rate, C-reactive protein, cerebrospinal fluid analysis and toxicology screen were within normal ranges. All cultures were negative. Electroencephalography and cranial magnetic resonance imaging were unremarkable. Hence we suspected clarithromycin as being responsible for the hallucinations, and it was discontinued. Over the following $24 \mathrm{~h}$ he completely recovered from the hallucinations. Psychiatric evaluation was not consistent with a primary psychiatric disorder. He has been observed monthly without any symptoms and treatment.

\section{Discussion}

Clarithromycin is a valuable macrolide antibiotic with a broader antimicrobial spectrum, oral bioavailability, high tissue penetration and a longer elimination half life than its parent compound erythromycin [3]. It is predominantly excreted by the liver at doses of $250 \mathrm{mg}$ twice daily. The renal excretion of clarithromycin and its 14-hydroxy metabolite can be as much as $30 \%$ at a dose of 500 mg twice daily. Peak serum concentrations may be achieved within $2 \mathrm{~h}$ after the onset of therapy in normal subjects. Higher levels can be reached in the functionally anephric patient with delayed elimination [2]. The most common adverse reactions involve gastrointestinal disturbances followed by hepatic cholestasis, allergic skin eruptions, ototoxicity with tinnitus and transient or irreversible deafness $[4,5]$. Lightheadedness, dizziness, confusion, insomnia and tinnitus have been reported in elderly patients treated with high-dose clarithromycin (1 g twice daily) [6]. Clarithromycin-associated hallucinations or psychotic behavior were attributed to drug interactions involving the capacity of macrolides to inhibit cytochrome $\mathrm{p} 450$, thereby increasing the plasma concentration of drugs given in combination [1, 7-9]. Probable clarithromycin-associated hallucination had also been reported in a 56-year-old man with chronic renal failure. In this case, poor renal function could possibly have facilitated the occurrence of this adverse reaction by altering the blood brain barrier, which could have increased the central nervous system concentrations of clarithromycin [2]. In 2002, Jiménez-Pulido et al. [1] reported that an adverse reaction to clarithromycin was the probable cause of the visual hallucinations according to the $\mathrm{Na}$ ranjo algorithm [10] in a 32-year-old woman receiving therapeutic doses of clarithromycin for upper respiratory tract infection. She had no medication other than clarithromycin or any systemic disorders as our patients, but unlike in this report our patients were not adults. In our cases, acute appearance of visual hallucinations shortly after the ingestion of clarithromycin with otherwise normal physical examination, normal laboratory investigations and imaging studies and gradual disappearance of symptoms once the clarithromycin therapy had been discontinued made us suspect clarithromycin as the agent responsible for the visual hallucinations.

\section{Conclusion}

This report highlights hallucinations due to therapeutic doses of clarithromycin therapy as a possible new side effect in children. 


\section{References}

-1 Jiménez-Pulido I, Navarro-Ruiz A, Sendra P, Martínez-Ramírez M, Garcia-Motos C, Montesinos-Ros A: Hallucinations with therapeutic doses of clarithromycin. Int J Clin Pharmacol Ther 2002;40:20-22.

-2 Steinman MA, Steinman TI: Clarithromycin-associated visual hallucinations in a patient with chronic renal failure on continuous ambulatory peritoneal dialysis. Am J Kidney Dis 1996;27:143-146.

3 Piscitelli SC, Danziger LH, Rodvold KA: Clarithromycin and azithromycin: new macrolide antibiotics. Clin Pharm 1992;11:137152 .
4 Periti P, Mazzei T, Mini E, Novelli A: Adverse effects of macrolide antibacterials. Drug Saf 1993;9:346-364.

5 Coulston J, Balaratnam N: Irreversible sensorineural hearing loss due to clarithromycin. Postgrad Med J 2005;81:58-59.

6 Wallace RJ Jr, Brown BA, Griffith DE: Drug intolerance to high-dose clarithromycin among elderly patients. Diagn Microbiol Infect Dis 1993;16:215-221.

7 Nightingale SD, Koster FT, Mertz GJ, Loss SD: Clarithromycin-induced mania in two patients with AIDS. Clin Infect Dis 1995;20: $1563-1564$.
-8 Cone LA, Sneider RA, Nazemi R, Dietrich EJ: Mania due to clarithromycin therapy in a patient who was not infected with human immunodeficiency virus. Clin Infect Dis 1996; 22:595-596.

$\checkmark 9$ Przybylo HJ, Przybylo JH, Todd Davis A, Coté CJ: Acute psychosis after anesthesia: the case for antibiomania. Paediatr Anaesth 2005;15:703-705.

10 Naranjo CA, Busto U, Sellers EM, Sandor P, Ruiz I, Roberts EA, Janecek E, Domecq C, Greenblatt DJ: A method for estimating the probability of adverse drug reactions. Clin Pharmacol Ther 1981;30:239-245. 